\title{
A comparison of the shrinkage of commercial bone cements when mixed under vacuum
}

F. N. K. Kwong, R. A. Power

From Glenfield Hospital, Leicester, England
F. N. K. Kwong, MRCSEd, Clinical Research Fellow R. A. Power, FRCSEd (Orth), Orthopaedic Surgeon Department of Orthopaedics Glenfield Hospital, University Hospitals of Leicester, Groby Road, Leicester LE3 9QP, UK.

Correspondence should be sent to Mr F. N. K. Kwong; e-mail: fcng@yahoo.com

C2006 British Editorial Society of Bone and Joint Surgery doi:10.1302/0301-620X.88B1. $16600 \$ 2.00$

$J$ Bone Joint Surg $[\mathrm{Br}]$ 2006;88-B:120-2

Received 7 April 2005;

Accepted after revision

13 July 2005

The outcome of a cemented hip arthroplasty is partly dependent on the type of cement which is used. The production of an interface gap between the stem and the cement mantle as a result of shrinkage of the cement, may be a factor involved. Palacos R, Palacos LV (both with gentamicin), CMW 1, CMW 2, CMW Endurance (CMWE) and Simplex were prepared under vacuum and allowed to cure overnight in similar cylinders. The next day this volume was determined by the displacement of water. Shrinkage varied between $3.82 \%$ and $7.08 \%$ with CMWE having the lowest and Palacos LV the highest. This could be a factor to consider when choosing a cement for a shape-closed stem.

The variation in outcome of cemented hip arthroplasties is dependent on several factors such as the design of the stem, the material, the cementing technique and the choice of cement used. Recently, greater emphasis has been placed on the importance of the cement-stem interface in aseptic loosening. ${ }^{1}$ There is a greater understanding of the relationship between cementing technique and design of the stem, as described by Huiskes, Verdonschot and Nivbrant. ${ }^{2}$ The latter authors borrowed concepts from engineering to categorise cemented stems according to the method by which they derive their stability. A shape-closed stem achieves this by its matching of shape with the femur but a force-closed stem is designed to subside within the cement mantle. ${ }^{2}$

With shape-closed stems, gaps at the cement-stem interface are thought to be a factor in the initiation of early loosening. When present at insertion such gaps may initiate debonding and it is thought that some debonded regions will expand until the whole interface is debonded. ${ }^{1,3}$ Debonding with a non-polished stem also leads to abrasion and the formation of debris at the cement-stem interface. ${ }^{1,3}$ In a model of a total hip arthroplasty, Wang, Franzen and Lidgren ${ }^{4}$ quantified the interface gap as being $10 \%$ to $15 \%$ of the whole interface area 12 hours after insertion of the stem.

Most of the interface gaps appear as a result of linear shrinkage of the cement around a stem. ${ }^{4-6}$ Cement shrinkage can also initiate damage within the cement mantle as a result of the residual stress occurring during curing. ${ }^{7,8}$

Although shrinkage of cement has been quantified in a few isolated commercial brands and its dynamic volume changes investigated, ${ }^{6,9}$ there is to date, to our knowledge, no published study of a direct comparison of cements. Our aim therefore was to investigate any difference in shrinkage, under the same standardised conditions of a range of polymethylmethacrylate cements in common use.

\section{Materials and Methods}

The cements studied are shown in Table I. All the experiments were done in the same laboratory under standardised conditions of temperature (mean $20.8^{\circ} \mathrm{C}$; SD $0.4 ; 20.0^{\circ} \mathrm{C}$ to $21.0^{\circ} \mathrm{C}$ ) and humidity (mean $35.2 \%$; SD $1.1 ; 34 \%$ to $36 \%$ ). The cements were stored for at least 12

Table I. Details of the cements studied

\begin{tabular}{lll}
\hline Cement & Manufacturer & Comments \\
\hline Palacos R (with gentamicin) & Schering-Plough Ltd (Welwyn Garden City, UK) & \\
Palacos LV (with gentamicin) & Schering-Plough Ltd & \\
CMW 1 Radio-opaque & Depuy International Ltd (Leeds, UK) & Low viscosity, also known as \\
CMW Endurance & Depuy International Ltd & Smartset MV \\
CMW 2 & Depuy International Ltd & Normally reserved for hand mixing \\
Simplex & Stryker-Howmedica Osteonics (Cork, Ireland) & \\
\hline
\end{tabular}


Table II. Details of shrinkage (\% initial volume; SD) in all the cements studied

\begin{tabular}{ll}
\hline Cement & Shrinkage \\
\hline CMW Endurance & $3.82(0.70)$ \\
CMW 1 & $5.05(0.50)$ \\
CMW 2 & $6.05(0.56)$ \\
Simplex & $5.93(0.36)$ \\
Palacos R & $6.20(0.36)$ \\
Palacos LV & $7.08(0.47)$ \\
\hline
\end{tabular}

hours in the laboratory before being used. For each mixing, one portion $(40 \mathrm{~g})$ of cement was used. Mixing was performed according to the manufacturers' recommendations, except for CMW 2 which is recommended for hand mixing only. However, since vacuum suction has an influence on shrinkage, ${ }^{6,10} \mathrm{CMW} 2$ was prepared in the same manner as the other cements for the purpose of our comparative study. For every cement, a minimum of six separate mixes was done. The mixing device used was a Summit Medical HiVac Syringe (Summit Medical, Bourton on the Water, Gloucestershire, UK) at a vacuum pressure of $550 \mathrm{~mm} \mathrm{Hg}$. The cement was injected into translucent polypropylene cylinders (Fisher Scientific, Loughborough, Leicestershire, UK) which had a volume of $35 \mathrm{ml}$.

The cement was then allowed to cure overnight at a temperature of $37^{\circ} \mathrm{C}$. Although the initial temperature of the femur just before injection of cement is lower than body temperature as a result of washing, ${ }^{10}$ the temperature inside the femur increases thereafter. In our study the temperature chosen for cement curing was body temperature. The next day, the plastic cylinders were sawn off at their lower portions and the cement blocks were gently punched out of the mould. This was a relatively easy task as a result of the overall volumetric shrinkage.

The volume of cement was determined by measuring the volume it occupied in water. The mass of water displaced, and hence the volume, was determined by weighing.

Statistical analysis. The means and SDs were calculated. A one-way analysis of variance (ANOVA) was used to assess whether there was any difference between the cements. This was followed by the post-hoc Gabriel's test for multiple comparisons. Analysis of data was performed using SPSS for Windows version 12.01 (SPSS Inc, Chicago, Illinois).

\section{Results}

Table II shows the results of shrinkage of the different brands of cement which were tested. Shrinkage varied between $3.82 \%$ and $7.08 \%$. CMWE had the lowest while Palacos LV had the highest shrinkage. Both are low-viscosity cements. There was a statistically significant difference in shrinkage between the different cements tested. A oneway ANOVA test showed the F ratio to be 15.7 ( $\mathrm{p}=0.001$ ). Gabriel's test was used to compare the shrinkage between all the groups and the results are shown in Table III.

\section{Discussion}

Our study has shown that, under standardised conditions, the shrinkage of polymethylmethacrylate cement is different in the different commecial preparations in current use. The figures for shrinkage in our study are in the range reported by previous studies. ${ }^{6,9}$ Theoretical calculations predicted that the molecular rearrangement occurring during polymerisation would produce a volumetric shrinkage of $7.6 \%{ }^{11}$

Espehaug et $\mathrm{al}^{12}$ reported the differences in the long-term outcome of a shape-closed stem, the Charnley stem, with different types of bone cement. It is possible that this difference in outcome was partly due to the difference in shrinkage reported in this study since those with high shrinkage may have poorer outcomes. However, it is difficult to correlate this material property with the long-term outcome because of the changes in formation of the cement over time and the relatively recent introduction of vacuum mixing 9 which is known to increase shrinkage. ${ }^{6,10,13}$ Vacuum mixing also affects other biomechanical properties of bone cement in a dose-dependent fashion. The porosity of bone cement when mixed is inversely proportional to the vacuum pressure. ${ }^{13,14}$ However, at a given vacuum pressure, any further decrease in porosity is likely to be offset by the production of cracks in the cement mantle. ${ }^{13}$

Polymer manufacturing techniques are available to eliminate polymerisation-induced shrinkage by controlled void formation or by including low-shrinkage polymer additives. However, attempts to change cement formulation, can be fraught with problems, as exemplified by the Boneloc cement. ${ }^{15,16}$

We would like to acknowledge Dr Guido Hermann from the Department of Engineering at the University of Leicester for advice; David Thompson for technical assistance; Summit Medical for their technical and material assistance; Foxtrot Charity for funding; and Stryker-Howmedica Osteonics and ScheringPlough Ltd for their material assistance.

Table III. Gabriel's test of comparison of shrinkage when the difference between the groups was statistically significant, the $p$ value is given; $p<0.05$

\begin{tabular}{lllllll}
\hline Cement & CMW E & CMW 1 & CMW 2 & Palacos R & Simplex & Palacos LV \\
\hline CMW E & & & 0.002 & 0.001 & 0.005 & 0.001 \\
CMW 1 & & & 0.034 & & 0.001 \\
CMW 2 & 0.002 & & & & \\
Palacos R & 0.001 & 0.034 & & & 0.029 & 0.029 \\
Simplex & 0.005 & & & & \\
Palacos LV & 0.001 & 0.001 & & & \\
\hline
\end{tabular}


The author or one or more of the authors have received or will receive benefits for personal or professional use from a commercial party related directly or indirectly to the subject of this article.

\section{References}

1. Verdonschot $\mathbf{N}$, Huiskes R. The effects of cement-stem debonding in THA on the long-term failure probability of cement. J Biomech 1997;30:795-802.

2. Huiskes R, Verdonschot N, Nivbrant B. Migration, stem shape, and surface finish in cemented total hip arthroplasty. Clin Orthop 1998;355:103-12.

3. Jasty M, Bragdon CR, Bragdon CR, et al. The initiation of failure in cemented femoral components of hip arthroplasties. J Bone Joint Surg [Br] 1991;73-B:551-8.

4. Wang JS, Franzen $\mathbf{H}$, Lidgren L. Interface gap after implantation of a cemented femoral stem in pigs. Acta Orthop Scand 1999;70:234-9.

5. Race A, Miller MA, Ayers K, Mann KA. Shrinkage of vacuum mixed cement causes interface gaps: gap distribution depends on stem surface finish. J Bone Joint Surg [Br] 2004;86-B(Supp I):9.

6. Gilbert JL, Hasenwinkel JM, Wixson RL, Lautenshlager EP. A theoretical and experimental analysis of polymerisation shrinkage of bone cement: a potential source of porosity. J Biomed Mater Res 2000;52:210-18.
7. Lennon AB, Prendergast PJ. Residuals stress due to curing can initiate damage in porous bone cement: experimental and theoretical evidence. J Biomech2002;35:311-21.

8. Roques A, Browne M, Taylor A, New A, Baker D. Quantitative measurement of the stresses induced during polymerisation of bone cement. Biomaterials 2004;25:4415-24.

9. Muller SD, Green SM, McCaskie AW. The dynamic volume changes of polymerising polymethylmethacrylate bone cement. Acta Orthop Scand 2002;73:684-7.

10. Jafri AA, Green SM, Partington PF, McCaskie AW, Muller SD. Pre-heating of components in cemented total hip arthroplasty. J Bone Joint Surg [Br] 2004;86-B: 1214-19.

11. Haas S, Brauer G, Dickson G. A characterisation of polymethylmethacrylate bone cement. J Bone Joint Surg [Am] 1975;57-A:380-91.

12. Espehaug B, Furnes O, Havelin LI, Engesaeter LB, Vollset SE. The type of cement and failure of total hip replacements. J Bone Joint Surg [Br] 2002;84-B:832-8.

13. Dunne NJ, Orr JF. The effects of porosity on acrylic bone cement shrinkage after polymerisation [abstract]. Procs European Society for Biomaterials Conference, 2001.

14. Wang JS, Franzen H, Jonsson E, Lidgren L. Porosity of bone cement reduced by mixing and collecting under vacuum. Acta Orthop Scand 1993:64:143-6.

15. Abdel-Kader KF, Allcock S, Walker DI, Chaudhry SB. Boneloc bone-cement: experience in hip arthroplasty during a 3-year period. J Arthroplasty 2001;16:811-19.

16. Gebuhr P, Stentzer K, Thomsen F, Levi N. Failure of total hip arthoplasty with Boneloc bone cement. Acta Orthop Belg 2000;66:472-6. 